\title{
Expression of the Transcription Factor Egr-1 in Pancreatic Acinar Cells Following Stimulation of Cholecystokinin or Gaq- Coupled Designer Receptors
}

\author{
Anke Kaufmann Oliver G. Rössler Gerald Thiel \\ Department of Medical Biochemistry and Molecular Biology, University of Saarland Medical Center \\ Homburg, Germany
}

\author{
Key Words \\ AR42J cells $•$ Cerulein $\bullet$ Egr-1 $\bullet$ Lentivirus $\cdot$ Pancreatitis $\bullet$ Designer receptor
}

\begin{abstract}
Backgound/Aims: The injection of cerulein, an analogue of the pancreatic secretagogue cholecystokinin (CCK), induces acute pancreatitis in mice that is accompanied by the synthesis of the transcription factor Egr-1. The signaling cascade that connects cerulein stimulation with enhanced Egr-1 biosynthesis was analyzed. Methods: AR42J rat pancreatic acinar cells were used as a model system to measure cerulein-induced Egr-1 biosynthesis. For comparison, the signaling cascade induced by activation of Gaq-coupled designer receptors with the designer drug clozapine- $N$-oxide (CNO) was investigated. Results: Stimulation of AR42J cells with cerulein induced a robust and transient biosynthesis of Egr-1. The signaling cascade connecting cerulein stimulation with Egr-1 gene expression required elevated levels of cytosolic $\mathrm{Ca}^{2+}$ and the activation of the protein kinases PKC, Raf and ERK, while expression of MKP-1 prevented Egr-1 biosynthesis in cerulein-stimulated AR42J cells. In addition, ternary complex factors are required to connect cerulein stimulation with enhanced transcription of the Egr-1 gene. Egr-1 biosynthesis induced in CNO-stimulated AR42J pancreatic acinar cells expressing Gaq-coupled designer receptors required identical signaling molecules, although subtle differences were observed in comparison to cerulein/CCK receptor signaling. Conclusion: We propose that overstimulation of the canonical Gaq-induced signaling pathway may be crucial for inducing acute pancreatitis.
\end{abstract}




\section{Introduction}

Cerulein, a ten amino acid oligopeptide derived from the skin of the Australian green tree frog (Litoria caerulea), is a cholecystokinin (CCK) analogue that activates both CCK1 and CCK2 receptors [1]. Injection of a supraphysiological concentration of cerulein is used in an animal model of acute pancreatitis, that is characterized by massive tissue damage of pancreatic acinar cells, a local pancreatic inflammation, and an infiltration of inflammatory cells into the pancreas. A premature activation of trypsinogen within pancreatic acinar cells is generally believed to be the initiating event for the tissue-damage of the pancreas [2].

This local inflammation of the pancreas is accompanied by the synthesis and release of proinflammatory chemokines and cytokines, including tumor necrosis factor- $\alpha$ (TNF $\alpha$ ), macrophage chemotactic protein (MCP-1), macrophage inflammatory protein-1 (MIP-1), and interleukin $1 \beta$ [3-6]. In addition, an upregulation of the intracellular adhesion molecule ICAM-1 has been reported [4]. Interestingly, the genes encoding TNF $\alpha$, MCP-1, MIP-1, and ICAM-1 have been described as target genes for the zinc finger transcription factor Egr-1 [7-14].

Egr-1 is regulated via its biosynthesis. In unstimulated cells, only low levels of Egr-1 are detectable. Upon stimulation by extracellular signaling molecules including growth factors, hormones, and neurotransmitters, the biosynthesis of Egr- 1 is induced $[15,16]$. Egr-1 couples extracellular signals with long-term responses by altering the gene expression pattern of Egr1 target genes. The fact that Egr-1 regulates expression of genes encoding proinflammatory chemokines and cytokines is the basis for the hypothesis that Egr-1 functions as a master switch during inflammation. The analysis of Egr-1-deficient mice revealed that inactivation of the Egr-1 gene diminished expression of mediators for vascular injury in an animal model of lung ischemia/reperfusion [9]. Egr-1 is synthesized in pancreatic acinar cells within 1-4 hours following induction of acute pancreatitis by cerulein injection [11, 14, 17]. Moreover, expression of inflammation-related gene products, including MCP-1, MIP-1 and ICAM-1, and lung inflammation were reduced in cerulein-treated Egr-1-deficient mice, linking Egr-1 activity with the severity of cerulein-induced acute pancreatitis $[11,14,17]$.

In this study, we have analyzed the signaling cascade leading to enhanced Egr-1 biosynthesis in cerulein-stimulated AR42J pancreatic acinar cells. Additionally, we have investigated the intracellular signaling cascade following stimulation of Gaq-coupled designer receptors in AR42J cells. The results show that both intracellular signaling cascades, induced by either stimulation of CCK receptors with cerulein or stimulation of Gaq-coupled designer receptors with CNO, require a rise of intracellular $\mathrm{Ca}^{2+}$, the activation of the protein kinases PKC, Raf, and ERK, and in the nucleus, the activation of the transcription factor Elk-1.

\section{Materials and Methods}

\section{Cell culture}

AR42J cells were kindly provided by Bernd Baumann, University of Ulm, Germany, and maintained in Dulbecco's modified Eagles medium supplemented with $10 \%$ heat inactivated fetal calf serum, penicillin $(100 \mathrm{U} / \mathrm{ml})$ and streptomycin $(100 \mu \mathrm{g} / \mathrm{ml})$ at $37^{\circ} \mathrm{C}$ in $5 \% \mathrm{CO}_{2}$. Cells were cultured in medium containing $0.05 \%$ fetal bovine serum for 24 hours prior to the stimulation. Stimulation with cerulein (10 nM, Sigma \# C9026, dissolved in water) was performed for 1 hour if not stated differently. Likewise, stimulation with either clozapine-N-oxide (CNO, $1 \mu \mathrm{M}$, Enzo Life sciences, \# NS-105-0005, dissolved in ethanol), EGF (10 nM, Promega, Mannheim, Germany, \# G5021, dissolved in $\mathrm{H}_{2} \mathrm{O}$ as a $100 \mu \mathrm{g} / \mathrm{ml}$ stock solution), or phorbol 12-0-tetradecanoylphorbol-13-acetate (TPA, 50 ng/ml, Calbiochem \# 524400-1, dissolved in DMSO) was perfomed for 1 hour in medium containing $0.05 \%$ fetal bovine serum. Cells were pre-incubated for 2 hours

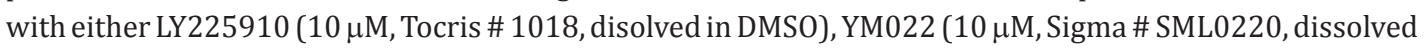
in DMSO), or lorglumide (10 $\mu \mathrm{M}$, Sigma \# L109, dissolved in methanol) prior to the stimulation. Cells were pre-incubated for 3 hours with either bisindolylmaleimide III (2 $\mu \mathrm{M}$, ENZO \# ALX-270-051, dissolved in

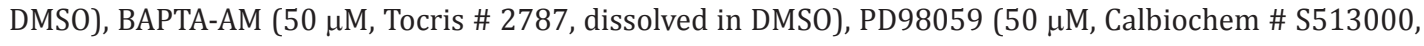


dissolved in DMSO), SP600125 (5 $\mu$ M, ENZO \# BML:EI305-0010, dissolved in DMSO), or SB203580 (5 $\mu$ M, ENZO \# BML-EI286-0001, dissolved in DMSO) prior to the stimulation with either cerulein, EGF or TPA. Incubation of the cells with TPA $(50 \mathrm{ng} / \mathrm{ml})$ to reduce PKC expression was performed for twenty-four hours. 4-Hydroxytamoxifen (4-OHT, Sigma \# H7904) was dissolved in ethanol and used at a concentration of 0.25 $\mu \mathrm{M}$.

Retroviral gene transfer

Plasmid pBabepuro3 $\triangle \mathrm{B}-\mathrm{Raf}: \mathrm{ER}$, encoding a B-Raf/estrogen receptor fusion protein, was kindly provided by Martin McMahon, UCSF, San Francisco, USA [18]. The packaging cell line Phoenix-Ampho was obtained from Gary Nolan, Stanford University, USA. Phoenix-Ampho cells were transfected with the retroviral vector using the calcium coprecipitation procedure. Retroviral infection of AR42J cells was performed as described [19]. AR42J cells were selected with $0.25 \mu$ g puromycin $/ \mathrm{ml}$. Mass pools of stable transfectants were selected and used for all experiments in order to eliminate the possibility of specific clonal effects.

\section{Lentiviral gene transfer}

The lentiviral transfer vectors pFUW-R $\alpha q$, pFUW-REST/Elk-1 $\Delta \mathrm{C}$, pFUW-MKP-1, pFUW-MKP-5, and pFUWmycDA-Raf1 have been described previously [20-23]. The viral particles were produced by triple transfection of HEK293T/17 cells with the gag-pol-rev packaging plasmid, the env plasmid encoding VSV glycoprotein, and the transfer vector [24].

Preparation of cell extracts and Western Blot analysis

Nuclear extracts and whole cell extracts were prepared as previously described [25]. $20 \mu \mathrm{g}$ of nuclear proteins were separated by SDS-PAGE and the blots were incubated with an antibody directed against Egr-1 (Santa Cruz, Heidelberg, Germany, \# sc-189). An antibody directed against HDAC1 (Santa Cruz, Heidelberg, Germany, \# sc-81598) was used as a loading control as described recently [23, 26]. To detect FLAG-tagged proteins, we used the M2 monoclonal antibody directed against the FLAG epitope (SigmaAldrich, Steinheim, Germany, \# F3165), at 1:3000 dilution in TBST. Antibodies against the myc epitope were prepared from CRL-1729 hybridomas, purchased from ATCC. Immunoreactive bands were detected via enhanced chemiluminescence as described $[23,26]$.

$R T-P C R$

Total RNA isolated from AR42J cells was purified with the Qiagen RNeasy Plus Micro Kit (Cat.No: 74034). RNA samples were reverse transcribed into cDNA with RevertAid M-MuLV RT (Fermentas) in the presence of RNase Inhibitor (Fermentas). The PCR conditions were: one cycle at $95^{\circ} \mathrm{C}$ for $5 \mathrm{~min}, 32$ amplification cycles, each cycle consisted of denaturation at $95^{\circ} \mathrm{C}$ for $30 \mathrm{sec}$, primer annealing at $59^{\circ} \mathrm{C}$ for $30 \mathrm{sec}$, and extension at $72^{\circ} \mathrm{C}$ for $30 \mathrm{sec}$. RT-PCR was performed using the primer pairs $5^{\prime}$-GAC TCG GTA GCC CAT TAC AAT C-3'/5'-ACT TTC CCA AGT AGG TCA CGG-3' (Egr-3) and 5'-TTG TGA TGG GTG TGA ACC AC3'/5'-GTC TTC TGG GTG GCA GTG AT-3' (GAPDH).

\section{Results}

Biosynthesis of Egr-1 in cerulein-treated AR42J cells

AR42J pancreatic acinar cells were serum-starved for 24 hours and then incubated with cerulein for 1 hour. Egr-1 immunoreactivity was virtually undetectable in the absence of stimulation. In contrast, cerulein treatment strikingly increased the biosynthesis of Egr-1 (Fig. 1A). The expression of HDAC1 was analyzed as a loading control. An analysis of the time frame revealed that Egr-1 biosynthesis was transient with a peak expression occurring 1 hour following stimulation (Fig. 1B). Cerulein treatment also increased the level of Egr-3 mRNA (Fig. 1C). However, we did not detect Egr-3 by Western blot analysis, indicating that the Egr-3 levels are low in AR42J cells. We therefore focused our studies on the stimulusinduced biosynthesis of Egr-1. 
Fig. 1. Stimulation of AR42J pancreatic acinar cells with cerulein induces the biosynthesis of Egr-1. (A) AR42J cells were serum-starved for twenty-four hours and then treated with cerulein (10 nM) for 1 hour. Nuclear extracts were prepared and subjected to Western blot analysis. The blots were incubated with an antibody directed against Egr-1. As a control, expression of histone deacetylase-1 (HDAC1) was analyzed. (B) Serum-starved AR42J cells were treated with cerulein (10 nM) for $1,3,5,8$ or 24 hours as indicated and expression of Egr-1 was analyzed by immunoblotting. (C) Stimulation of AR42J pancreatic acinar cells with cerulein upregulates Egr-3 mRNA levels. AR42J cells were stimulated with cerulein for 1 hour. Total RNA was isolated, the mRNA reverse transcribed and the cDNA analyzed by PCR using primers to detect Egr-3 and GAPDH mRNAs.

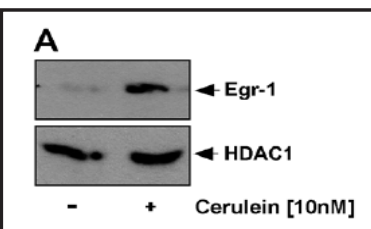

B

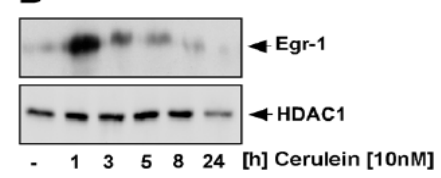

C
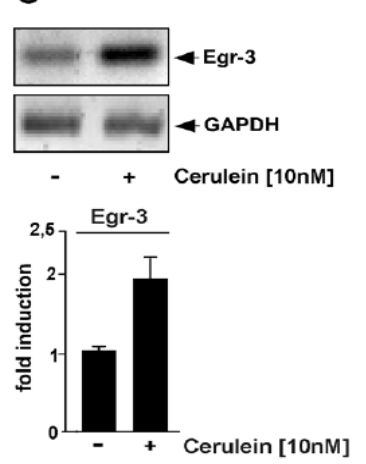

D

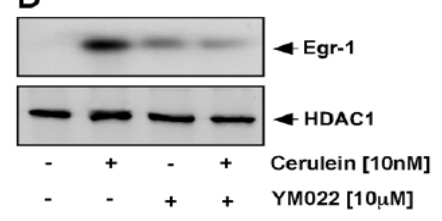

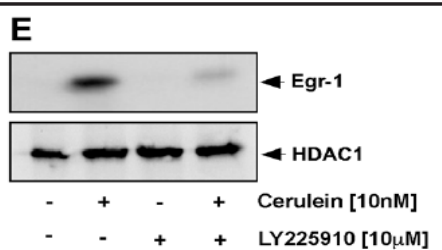

F

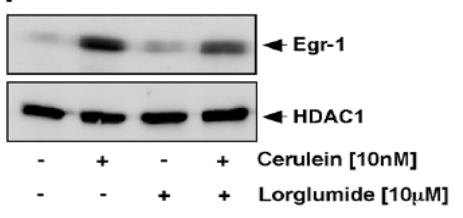

G

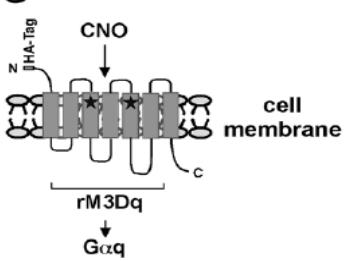

H

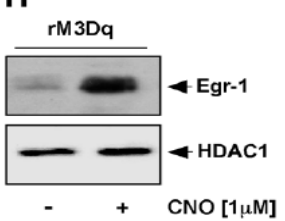

For quantification, the Egr-3 signal was normalized to the GAPDH signal and the fold-stimulation was calculated. (D-F) Serum-starved AR42J cells were preincubated with the CCK receptor inhibitors YM022 (10 $\mu \mathrm{M})(\mathrm{D})$, LY225910 $(10 \mu \mathrm{M})(\mathrm{E})$, or lorglumide $(10 \mu \mathrm{M})(\mathrm{F})$ for 2 hours. Cells were then stimulated with cerulein $(10 \mathrm{nM})$ for 1 hour in the presence of the inhibitors. Nuclear extracts were prepared and analyzed by immunoblotting. (G). Schematic representation of the Gaq-coupled designer receptor rM3Dq. The backbone is the rat M3 muscarinic acetylcholine receptor. Two point mutations (Y148C and A238G) were introduced into the orthosteric binding pocket of the natural ligand within the third and fifth transmembrane domain to prevent binding of acetylcholine to the designer receptor. Gaq-coupled designer receptors were specifically activated by clozapine-N-oxide (CNO). (H) Induction of Egr-1 biosynthesis in CNO-stimulated AR42J cells expressing Gaq-coupled designer receptor. Cells were infected with a lentivirus encoding the Gaq-coupled designer receptor. The infected cells were cultured for twenty-four hours in medium containing $0.05 \%$ serum. Stimulation with CNO $(1 \mu \mathrm{M})$ for 1 hour was performed with medium containing $0.05 \%$ serum. Nuclear extracts were prepared and subjected to Western blot analysis using an antibody directed against Egr-1. Each experiment illustrated here and in all subsequent figures was repeated at least twice with consistent results.

Cerulein-induced biosynthesis of Egr-1 in AR42J cells requires CCK2 receptors

AR42J cells express both CCK1 and CCK2 receptors [27]. Cerulein binds and activates both CCK receptors [1]. We used a pharmacological strategy to investigate the impact of both CCK1 and CCK2 receptors on cerulein-induced upregulation of Egr-1 expression. AR42J 
Fig. 2. Chelation of intracellular $\mathrm{Ca}^{2+}$ attenuates expression of Egr-1 in cerulein-stimulated AR42J pancreatic acinar cells. AR42J cells (A, C) or AR42J cells expressing Gaq-coupled designer receptors (B) were preincubated for 3 hours with the calcium chelator BAPTA-AM $(50 \mu \mathrm{M})$. Cells were stimulated with either cerulein $(10 \mathrm{nM})(\mathrm{A})$, CNO $(1 \mu \mathrm{M})(\mathrm{B})$, or EGF (10 nM) (C) for 1 hour. Nuclear extracts were prepared and subjected to Western blot analysis. The blots were incubated with an antibody directed against Egr-1.

cells were preincubated with the CCK2 inhibitors YM022 or LY225910, or with the CCK1 inhibitor lorglumide. Figs 1D-F show that both CCK2 inhibitors reduce expression of Egr-1 in cerulein-stimulated AR42J cells, while lorglumide did not interfere with upregulation of Egr-1 as a result of cerulein stimulation.

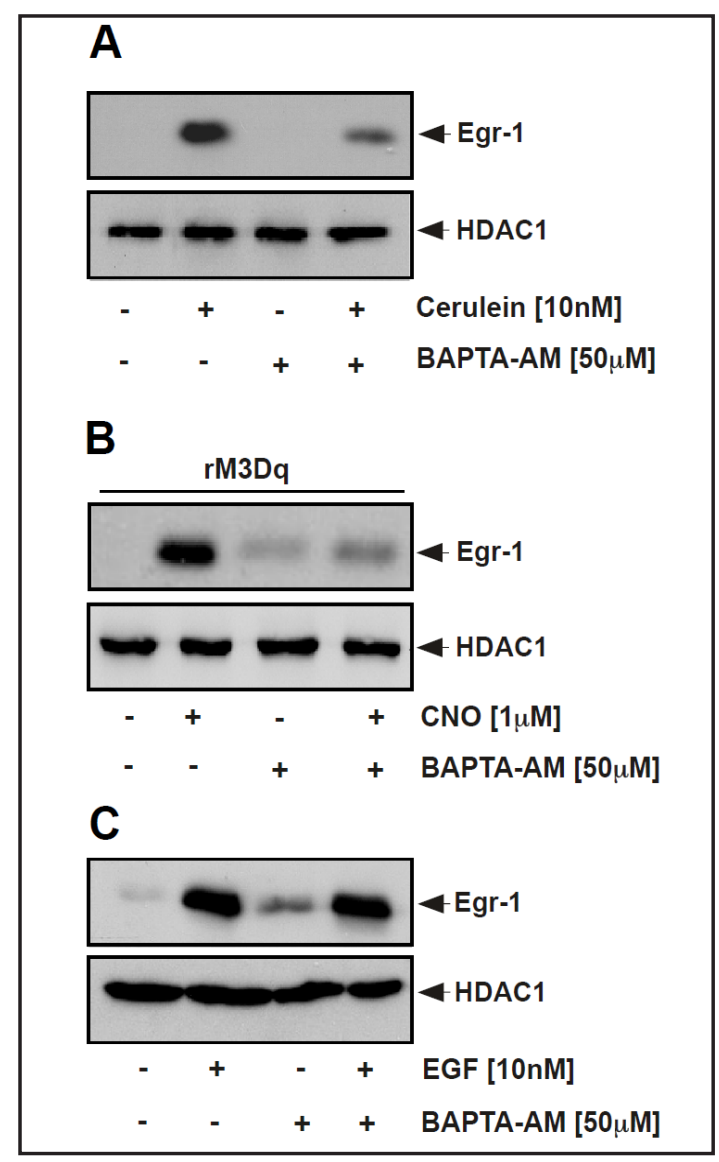

Biosynthesis of Egr-1 in AR42J cells following stimulation of Gaq-coupled designer receptors with a designer drug

CCK receptors are G-protein coupled receptors that activate phospholipase $\mathrm{C}$ via the $\mathrm{G}$ protein Gaq. For comparison, we expressed a Gaq-coupled designer receptor in AR42J cells (Fig. 1G) and stimulated the cells with the designer drug clozapine-N-oxide (CNO). Fig. 1H shows that stimulation of Gaq-coupled designer receptors triggered an upregulation of Egr1 in pancreatic AR42J cells.

Role of $\mathrm{Ca}^{2+}$ ions in cerulein-induced Egr-1 biosynthesis in AR42J pancreatic acinar cells

Stimulation of Gaq-coupled receptors leads to the activation of phospholipase C, the generation of $\mathrm{IP}_{3}$ and the release of $\mathrm{Ca}^{2+}$ ions into the cytosol via stimulation of ionotropic $\mathrm{IP}_{3}$ receptors of the endoplasmic reticulum. We assessed the importance of elevated $\left[\mathrm{Ca}^{2+}\right]_{\mathrm{i}}$ for the signaling cascade connecting cerulein-induced CCK2 receptor activation with Egr-1 gene transcription. Prior to stimulation with cerulein, AR42J cells were incubated in the presence or absence of BAPTA-AM, the cell-permeable acetoxymethylester of the cytosolic $\mathrm{Ca}^{2+}$ chelator BAPTA. When the cerulein-induced elevation of $\left[\mathrm{Ca}^{2+}\right]_{\mathrm{i}}$ was precluded by the preincubation with BAPTA-AM, the stimulus-induced upregulation of Egr-1 was reduced (Fig. 2A). Likewise, preincubation of the cells with BAPTA-AM interfered with the Egr-1 biosynthesis in CNO-stimulated AR42J cells expressing Gaq-coupled designer receptors (Fig. 2B). As a negative control, we stimulated the cells with EGF. Chelating intracellular $\mathrm{Ca}^{2+}$ with BAPTA-AM had no effect on the EGF-induced signaling cascade leading to Egr-1 expression (Fig. 2C).

PKC connects Gaq-coupled receptor activation with Egr-1 biosynthesis

The rise in intracellular $\left[\mathrm{Ca}^{2+}\right]$ following stimulation of Gaq-coupled receptors may activate protein kinase C (PKC), as shown in different cell types $[28,29]$. Stimulation of AR42J 


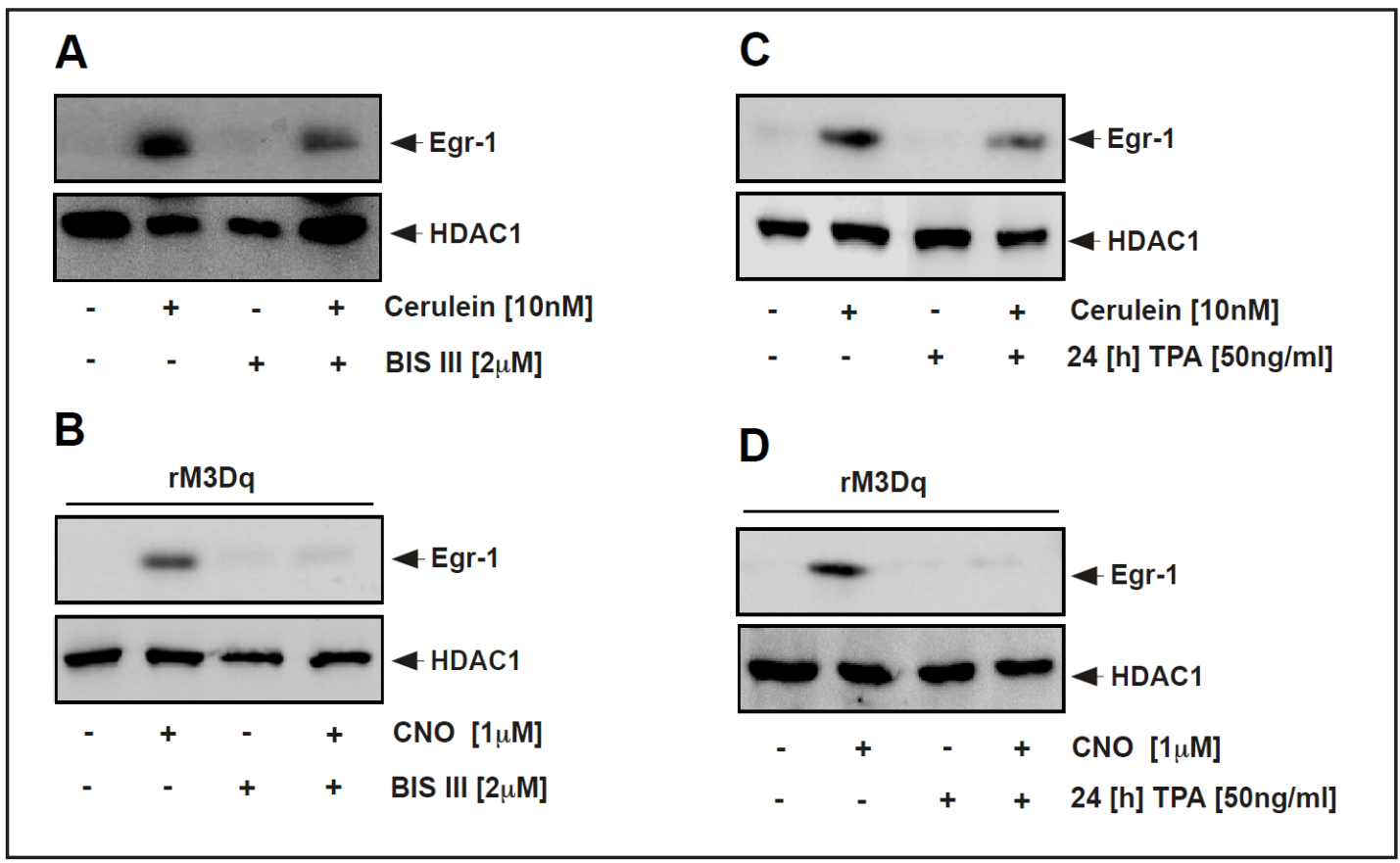

Fig. 3. Cerulein-regulated expression of Egr-1 requires the activation of PKC. AR42J cells (A) or AR42J cells expressing Gaq-coupled designer receptors (B) were preincubated with either vehicle or the PKC inhibitor bisindolylmaleimide III $(2 \mu \mathrm{M})$ for 3 hours as indicated. Cells were stimulated with either cerulein (10 nM) (A), or CNO $(1 \mu \mathrm{M})(\mathrm{B})$ for 1 hour. Nuclear extracts were prepared and subjected to Western blot analysis. The blots were incubated with an antibody directed against Egr-1. (C, D) AR42J cells (C) or AR42J cells expressing Gaq-coupled designer receptors (D) were preincubated with the phorbol ester TPA (50 ng/ml) for 24 hours to reduce expression of PKC. Cells were stimulated with either cerulein (10 nM) (C), or CNO (1 $\mu \mathrm{M}$ ) (D) for 1 hour. Nuclear extracts were prepared and subjected to Western blot analysis. The blots were incubated with an antibody directed against Egr-1.

cells with CCK has been shown to activate PKC [27]. To assess the involvement of PKC in the signaling cascade connecting cerulein stimulation with enhanced Egr-1 expression, we incubated AR42J cells with the protein kinase C inhibitor bisindolylmaleimide III, before the cells were stimulated with cerulein. Fig. 3A shows that the upregulation of Egr-1 expression was reduced under these conditions. This compound also blocked Egr-1 expression in CNOstimulated AR42J cells expressing Gaq-coupled designer receptors (Fig. 3B). Furthermore, AR42J cells were incubated with TPA for twenty-four hours to downregulate diacylgycerolregulated protein kinase $\mathrm{C}$ isoforms by promoting proteolytic degradation as described [27]. Figs. 3C and 3D reveal that prolonged treatment with TPA interfered with the cerulein or CNO-induced signaling pathway leading to the biosynthesis of Egr-1. Thus, these data support the conclusion that PKC activation is required to connect stimulation of the cells with enhanced Egr-1 biosynthesis.

Activation of Raf is essential for the induction of Egr-1 biosynthesis in AR42J cells stimulated with cerulein

Elevated intracellular $\mathrm{Ca}^{2+}$ concentrations as a result of stimulation of Gaq-coupled receptors trigger an activation of ERK, a crucial factor for the induction of Egr-1 biosynthesis in many cell types [21, 30-32]. In pancreatic acinar cells, CCK stimulation activates ERK [33]. The connection between an elevated $\mathrm{Ca}^{2+}$ concentration and the activation of the ERK signaling pathway is accomplished by PKC, that in turn, activates the protein kinase Raf. To demonstrate the importance of Raf in activating Egr-1 biosynthesis, we generated a AR42J 
Fig. 4. Essential role of Raf in connecting cerulein stimulation with enhanced Egr-1 transcription in AR42J pancreatic acinar cells. (A) Schematic representation of the modular structure of B-Raf and $\Delta \mathrm{B}$ Raf:ER. The functional domains of B-Raf (CR3, CR2, and CR1) are depicted. Fusion of the catalytic CR3 domain to the hormone binding domain of the estrogen receptor generates the $\Delta \mathrm{B}$ Raf:ER fusion protein. (B) Activation of B-Raf signaling

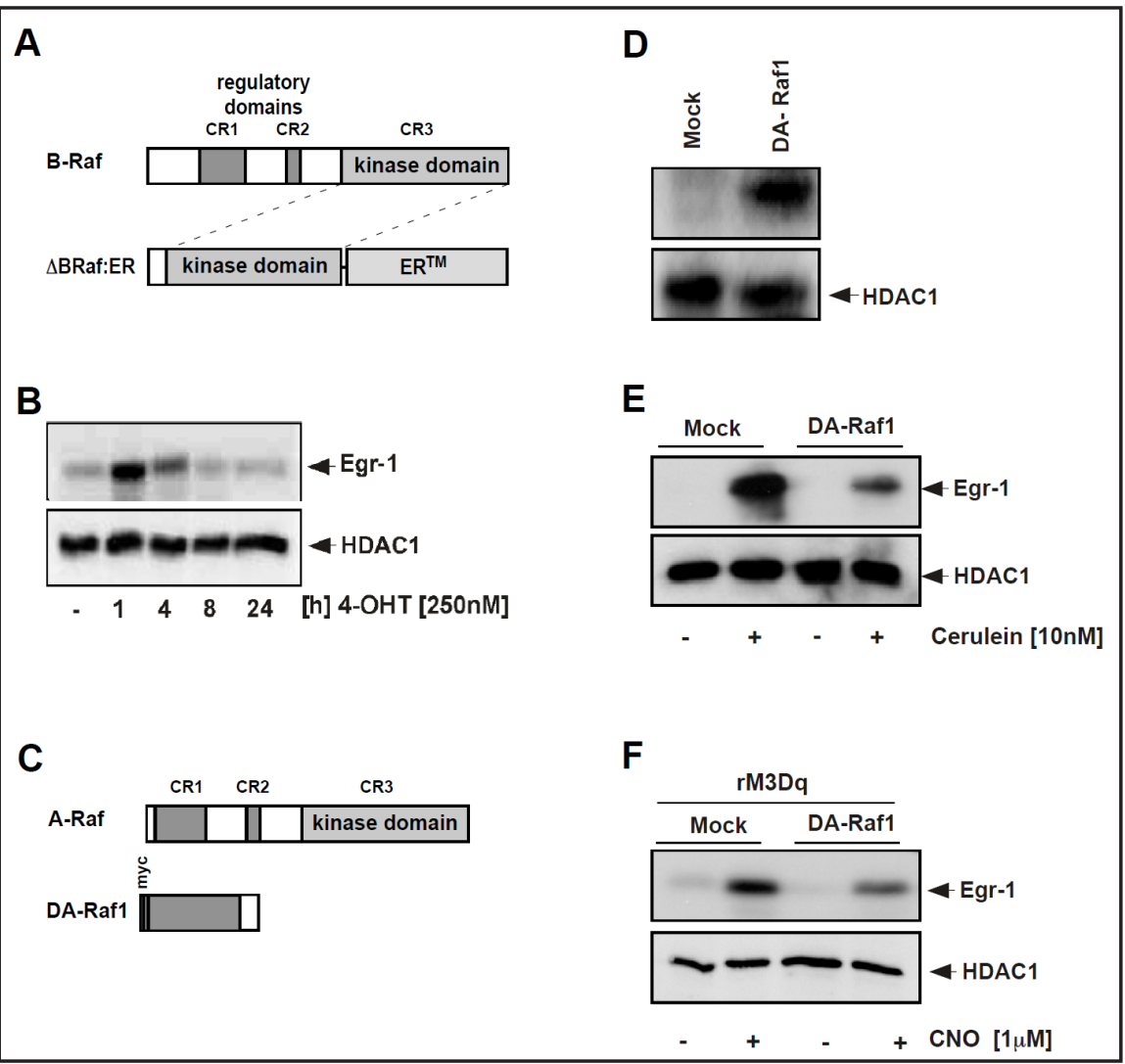

pathway in AR42J cells via expression of a $\triangle B$-Raf/estrogen receptor fusion protein triggers the biosynthesis of Egr-1. AR42J- $\Delta B$-Raf:ER cells were serum-starved for twenty-four hours. Cells were stimulated with 4-OHT (250 nM) as indicated. (C) Modular structure of A-Raf and DA-Raf1. (D) Expression of DA-Raf1 in lentiviral-infected AR42J cells was detected using an antibody against the N-terminal myc-tag. (E) AR42J cells were infected with a lentivirus encoding DA-Raf1. As a control AR42J cells were infected with lentiviral stocks prepared with the lentiviral transfer vector pFUW (mock). Cells were cultured for twenty-four hours in medium containing $0.05 \%$ serum. Stimulation was performed with medium containing cerulein (10 nM). Nuclear extracts were prepared and subjected to Western blot analysis. The blots were incubated with antibodies directed against either Egr-1, or HDAC1 as indicated. (F) AR42J cells were infected with a lentivirus encoding DA-Raf1. As a control AR42J cells were infected with lentiviral stocks prepared with the lentiviral transfer vector pFUW (mock). In addition, cells were infected with a lentivirus encoding Gaq-coupled designer receptors. Cells were cultured for twenty-four hours in medium containing $0.05 \%$ serum. Stimulation was performed with medium containing CNO $(1 \mu \mathrm{M})$. Nuclear extracts were prepared and analyzed by immunoblotting using antibodies directed against either Egr-1 or HDAC1.

cell line expressing a $\Delta \mathrm{B}$-Raf/estrogen receptor fusion protein, a conditionally active form of B-Raf. The modular structure of B-Raf and $\triangle \mathrm{B}$-Raf:ER is schematically depicted in Fig. 4A. B-Raf contains two regulatory domains (CR1, CR2) and a catalytic domain (CR3). Expression of the catalytic domain of B-Raf as a fusion protein with the ligand binding domain of the murine estrogen receptor (ER) maintains the protein kinase in an inactive state in the absence of hormone. Addition of hormone leads to an enhancement of B-Raf kinase activity [34]. Fig. 4B shows that stimulation of AR42J cells expressing the $\Delta B$-Raf/estrogen fusion protein with 4-OHT induced the biosynthesis of Egr-1 (Fig. 4B).

To corroborate these data and prove the role of Raf in cerulein-treated AR42J cells, loss-of-function experiments were performed using genetic and pharmacological tools: First, we inhibited Raf via expression of a dominant-negative antagonist of the Ras/MEK/ ERK1/2 pathway. Second, we treated AR42J cells with PD98059, a compound that inhibits 
Fig. 5. Essential role of ERK in stimulus-transcription coupling mediated by cerulein in AR42J pancreatic acinar cells. AR42J cells (A, C) or AR42J cells expressing Gaq-coupled designer receptors (B) were preincubated with PD98059 $(50 \mu \mathrm{M})$ for 3 hours. Cells were stimulated with either cerulein (10 nM) (A), CNO $(1 \mu \mathrm{M})(\mathrm{B})$, or EGF $(10 \mathrm{nM})(\mathrm{C})$ for 1 hour. Nuclear extracts were prepared and subjected to Western blot analysis. The blots were incubated with an antibody directed against Egr-1. (D, E) AR42J cells were preincubated with either SB203580 $(5 \mu \mathrm{M})$, or SP600125 $(5 \mu \mathrm{M})$ for 3 hours. Cells were stimulated with cerulein (10 $\mathrm{nM}$ ) for 1 hour.

phosphorylation of the MAP kinase kinase by Raf. Fig. 4C shows the modular structure of DA-Raf1, a splicing isoform of A-Raf that functions as an antagonist of the Raf/MEKERK1/2 signaling pathway [35]. Cellular proteins of mock-infected AR42J cells or cells infected with a myc-tagged DA-Raf1 encoding lentivirus were fractionated by SDS-PAGE. The fusion protein was identified by Western blot analysis using an antibody targeting the myc epitope (Fig. 4D). Next, the functional implication of DA-Raf1 expression was assessed. The results show that expression of DA-Raf1 significantly reduced the upregulation of Egr-1 expression in cerulein-stimulated AR42J cells (Fig. 4E). Likewise, DA-Raf1 reduced the expression of Egr-1 in CNO-stimulated AR42J cells expressing Gaq-coupled designer receptors (Fig. 4F). In addition, preincubation of the cells with PD98059 $(50 \mu \mathrm{M}$, as suggested [36]) efficiently blocked the upregulation of Egr-1 in cerulein-stimulated AR42J cells, or in CNO-stimulated AR42J cells expressing a Gaq-coupled designer receptor (Figs. 5A, B). Incubation of AR42J cells with PD98059 also blocked the upregulation of Egr-1 in

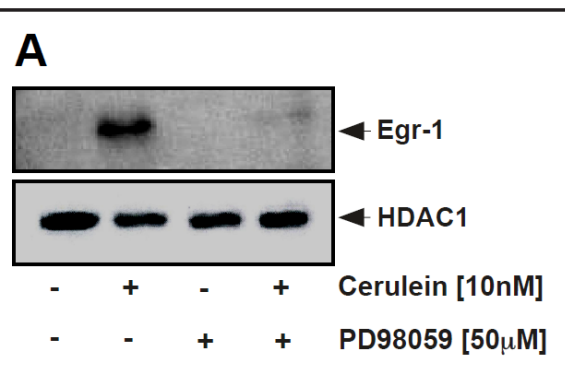

B
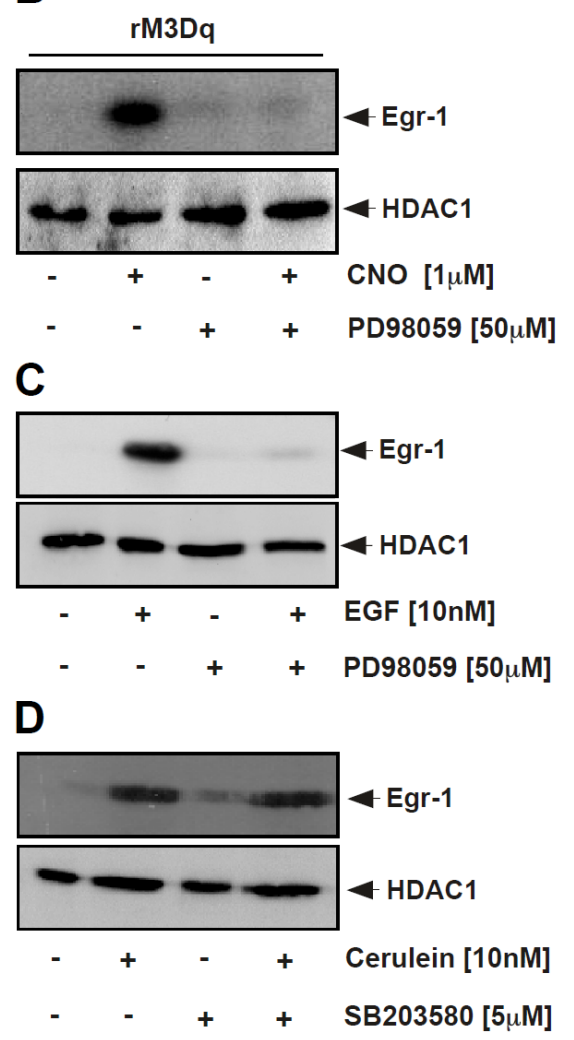

E

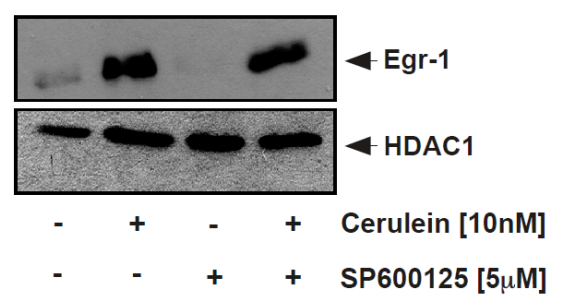

EGF-treated cells (Fig. 5C). In contrast, the MAP kinase inhibitors SB203580 or SP600125, described as p38 or JNK inhibitors, did not interfere with the upregulation of Egr-1 following stimulation of AR42J cells with cerulein (Figs. 5D and E).

\section{Overexpression of MKP-1 prevents cerulein-induced Egr-1 expression}

MKP-1, the enzyme that dephosphorylates ERK in the nucleus, is synthesized in different cell types following ERK activation, suggesting that MKP-1 is part of a negative feedback loop that inactivates ERK $[22,29,37,38]$. Fig. 6A shows that the biosynthesis of Egr-1 was impaired in cerulein-stimulated AR42J cells that had been infected with a MKP-1 encoding lentivirus. These data indicate that active ERK in the nucleus was required within 


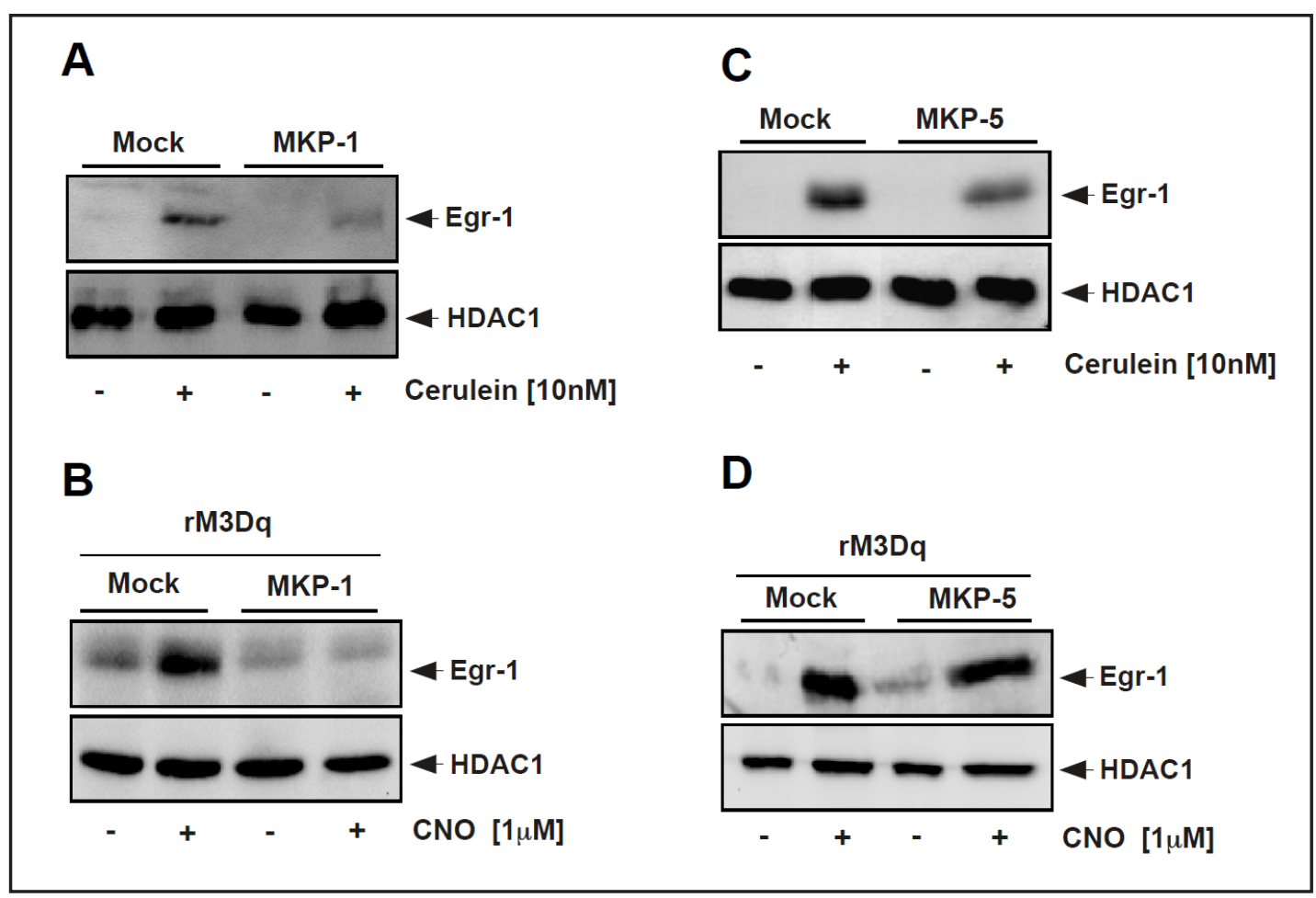

Fig. 6. Expression of MKP-1 attenuates cerulein-induced upregulation of Egr-1 in AR42J pancreatic acinar cells. (A, C) AR42J cells were infected with a lentivirus encoding either MKP-1 (A) or MKP-5 (C). As a control AR42J cells were infected with lentiviral stocks prepared with the lentiviral transfer vector pFUW (mock). Serum-starved cells were stimulated with cerulein (10 $\mathrm{nM}$ ) for 1 hour. (B, D) AR42J cells were infected with either a lentivirus encoding MKP-1 or MKP-5, or, as a control, with lentiviral stocks prepared with the lentiviral transfer vector pFUW (mock). In addition, cells were infected with a lentivirus encoding Gaq-coupled designer receptors. Cells were cultured for twenty-four hours in medium containing $0.05 \%$ serum. Stimulation was performed with medium containing CNO $(1 \mu \mathrm{M})$. Nuclear extracts were prepared and analyzed by immunoblotting using antibodies directed against either Egr-1 or HDAC1.

the signaling cascade. Likewise, Egr-1 biosynthesis was attenuated in CNO-stimulated AR42J cells expressing Gaq-coupled designer receptors (Fig. 6B). In contrast, expression of MKP-5, the phosphatase that dephosphorylates and inactivates p38 and c-Jun N-terminal protein kinases in the nucleus, did not significantly interfere with the upregulation of Egr-1 following stimulation of CCK2 receptors with cerulein or Gaq-coupled designer receptors with CNO in AR42J cells (Figs. $6 \mathrm{C}$ and D).

Suppression of ternary complex factor activity impairs Egr-1 biosynthesis in ceruleinstimulated AR42J pancreatic acinar cells

The serum response elements (SRE) within the Egr-1 promoter function as binding sites for the serum response factor (SRF) and ternary complex factors (TCF). TCFs are proteins that contact DNA and also bind to SRF. We assessed the impact of ternary complex factor activation on the regulation of Egr-1 biosynthesis in cerulein-stimulated AR42J cells. To overcome the problem associated with redundancy of functions between ternary complex factors [39], we expressed a dominant-negative mutant of the ternary complex factor Elk-1, termed REST/Elk-1 $\Delta$ C. The mutant retains the DNA-binding and SRF interaction domain, but lacks the C-terminal activation domain of Elk-1 (Fig. 7A). REST/Elk-1 $\Delta \mathrm{C}$ additionally contains the N-terminal repression domain of the transcriptional repressor REST [40], an immunological tag used for detection of the protein (FLAG epitope) and a nuclear localization 


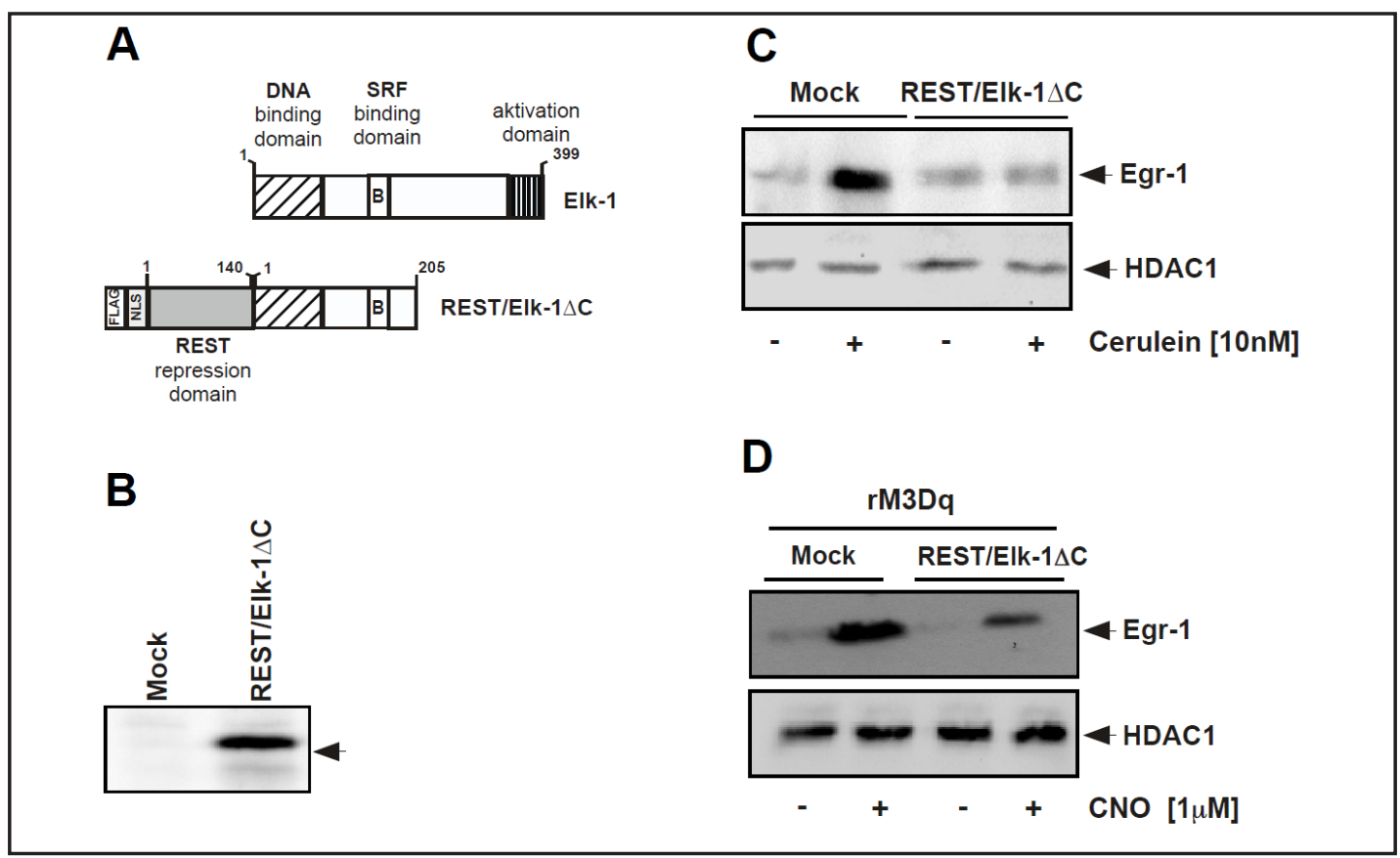

Fig. 7. Ternary complex factors play an essential role for inducing Egr-1 expression in cerulein-treated AR42J pancreatic acinar cells. (A) Schematic representation of the modular structure of the ternary complex factor Elk-1 and the dominant-negative mutant REST/Elk-1 $\Delta$ C. The mutant lacks the phosphorylation-regulated activation domain, but retains the DNA and SRF binding domains. The truncated Elk-1 was expressed as a fusion protein together with a transcriptional repression domain derived from the transcriptional repressor REST. (B) Western blot analysis of mock-infected AR42J cells or AR42J cells infected with a recombinant lentivirus encoding REST/Elk-1 $\Delta \mathrm{C}$. The Western blot was probed with an antibody against the FLAG-tag. (C) Expression of REST/Elk-1 $\Delta$ C blocks cerulein-induced upregulation of Egr-1 in AR42J cells. Cells were either mock-infected or infected with a recombinant lentivirus encoding REST/Elk-1 $\Delta$ C. Serum-starved cells were stimulated with cerulein (10 nM) for 1 hour. (D) AR42J cells were either mock-infected or infected with a recombinant lentivirus encoding REST/Elk-1 $\Delta$ C. In addition, cells were infected with a lentivirus encoding Gaq-coupled designer receptors. Serum-starved cells were stimulated with CNO $(1 \mu \mathrm{M})$ for 1 hour. Nuclear extracts were prepared and subjected to Western blot analysis. The blot was incubated with an antibody directed against Egr-1. The antibody directed against HDAC1 was used as a loading control.

signal (NLS). Nuclear proteins of mock-infected AR42J cells or cells infected with a REST/Elk$1 \triangle \mathrm{C}$ encoding lentivirus were fractionated by SDS-PAGE. The fusion protein was identified by Western blot analysis using antibodies targeting the FLAG epitope. Fig. 7B shows that the REST/Elk-1 $\Delta \mathrm{C}$ fusion protein was synthesized as expected. Next, the functional implications of REST/Elk- $1 \Delta \mathrm{C}$ expression were assessed. The results show that REST/Elk-1 $\Delta \mathrm{C}$ blocked the biosynthesis of Egr-1 in cerulein-stimulated AR42J cells (C). Likewise, stimulation of Gaq-coupled designer receptors did not induce an upregulation of Egr-1 in AR42J cells expressing the dominant-negative mutant of Elk-1, REST/Elk-1 $\Delta \mathrm{C}$ (D). Thus, TCF activity is essential for upregulating Egr-1 gene transcription as a result of CCK receptor or Gaqcoupled designer receptor stimulation.

\section{Discussion}

Acute pancreatitis is an inflammatory disease that is life-threating in its severe form. The disease is accompanied by an activation of pancreatic enzymes in the pancreas and a subsequent exaggerated inflammatory response that is not limited to the pancreas but 
includes other tissues, most importantly the lung. The precise molecular events leading to the induction of acute pancreatitis have not yet been elucidated in detail. There are several animal models of experimental pancreatitis. One of the best-characterized and frequently used model is based on the injection of the CCK analogue cerulein into mice. This treatment results in a pancreatitis phenotype including trypsin activation in the pancreas, cytoplasmic vacuolization, edema formation, and infiltration of inflammatory cells into the pancreas. The fact that expression of the inflammatory transcription factor Egr-1 has been causally connected with acute pancreatitis $[11,14,17]$ provided the stimulus for investigating the signal transduction following cerulein stimulation of pancreatic acinar cells. The objective of this study was the elucidation of the cerulein-induced signaling cascade in AR42J pancreatic acinar cells leading to enhanced expression of Egr-1.

Cerulein binds to both CCK1 and CCK2 receptors that both are Gaq-coupled receptors [41]. However, G protein-coupled receptors may couple to more than one G protein with the result that distinct signaling pathways are activated. In cardiomyocytes, for instance, the

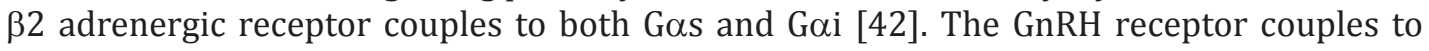

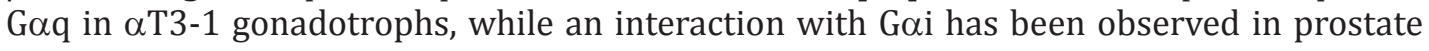
cancer cells [43], indicating that the G protein specificity of a particular receptor may be cell type-dependent. Likewise, coupling of CCK2 receptors to pertussis-toxin sensitive Gi/o has been observed in parvalbumin-positive GABAergic basket cells of the hippocampus, while in pyramidal cells the canonical Goq-pathway is induced by CCK via activation of CCK2 receptors [44]. Therefore, we expressed for comparison a Gaq-coupled designer receptor exclusively activated by designer drug (DREADD) in AR42J pancreatic acinar cells. These designer $\mathrm{G}$ protein-coupled receptors have been developed to specifically activate a particular G protein induced signaling cascades. The backbone for the Gaq-coupled designer receptor is the rat M3 muscarinic acetylcholine receptor. Binding of acetylcholine to the designer receptor is prevented by introducing two point mutations (Y148C and A238G) into the orthosteric binding pocket of the natural ligand within the third and fifth transmembrane domain of the receptor. This receptor lacks constitutive activity, is unresponsive to the endogenous ligand acetylcholine, but can be activated by the otherwise pharmacologically inert compound clozapine- $N$-oxide (CNO) $[45,46]$. The results of this study show that cerulein signals via the CCK2 receptor in AR42J cells to induce Egr-1 expression. Moreover, the data reveal that cerulein-stimulated CCK2 receptor signaling and CNO-induced Gaqcoupled designer receptor signaling activated identical intracellular signaling molecules, indicating that cerulein-induced pancreatitis relies on the overstimulation of the canonical Gaq-characterized signaling cascade.

Hallmarks of Gaq-induced signaling are the rise in intracellular $\mathrm{Ca}^{2+}$ and the subsequent activation of PKC. Pharmacological experiments confirm that both $\mathrm{Ca}^{2+}$ ions and phorbol ester responsive PKC isoforms are crucial for the signaling connecting CCK2 receptor activation with enhanced Egr-1 gene transcription. These data support the view that CCK2 receptor stimulation leads to an activation of the Goq-signaling pathway. In fact, activation of PKC $\varepsilon$ has been demonstrated to be crucial for CCK-mediated activation of ERK [27]. A comprehensive analysis of Gaq-coupled designer receptor signaling revealed that stimulation of this receptor type activates the protein kinases Raf and extracellular signalregulated protein kinase, as shown in studies analyzing $\mathrm{AT}_{1}$ angiotensin II receptors, M3 muscarinic acetylcholine receptors, gonadotropin releasing hormone receptors, calciumsensing receptors, and Gaq-coupled designer receptors [20, 21, 31, 47]. The data presented here show that both Raf and ERK are required to connect cerulein stimulation with Egr-1 gene transcription in AR42J pancreatic acinar cells.

The phosphorylated and activated ERK translocates into the nucleus and may change the transcriptional program by phosphorylating transcriptional regulatory proteins. In the nucleus, MKP-1 inactivates ERK via dephosphorylation and attenuates ERK regulated gene transcription [21, 22, 29, 38, 47, 48]. Expression of MKP-1, a dual-specific phosphatase that dephosphorylates and inactivates ERK in the nucleus, blocked the upregulation of Egr1 following stimulation of AR42J cells with cerulein, indicating that nuclear MAP kinase 
activity is essential for the signaling cascade connecting cerulein-induced CCK2 receptor stimulation with enhanced transcription of Egr-1.

A major nuclear substrate for ERK is Elk-1, a member of the Ets family of transcription factors. Elk-1 is an essential component of the serum response ternary complex that binds to DNA and to the serum response factor SRF. The transcriptional activity of Elk-1 depends on its phosphorylation-status. Elk-1 is phosphorylated by several protein kinases including ERK, leading to enhanced DNA binding, ternary complex formation and SRE-mediated transcription. Phosphorylation of Elk-1 connects the ERK signaling cascade with SREmediated gene transcription. The human Egr-1 promoter contains five SREs encompassing the consensus sequence $\mathrm{CC}[\mathrm{A} / \mathrm{T}]_{6} \mathrm{GG}$, known as the $\mathrm{CArG}$ box, and multiple binding sites for Elk-1 and other ternary complex factors adjacent to the CArG boxes. Transcriptional activation of Egr-1 is often preceded by an activation of Elk-1, indicating that the SREs within the Egr-1 promoter mediate signal-induced activation of Egr-1 gene transcription. To assess the necessity of ternary complex factor activation within the signaling cascade connecting cerulein stimulation with enhanced Egr-1 gene transcription, we performed loss-of-function experiments. Genetic inactivation of Elk-1 or other ternary complex factors in transgenic mice revealed minimal changes of the phenotype $[39,49,50]$, suggesting that functional redundancy may exist. Therefore, we have expressed a dominant negative version of Elk-1. Due to its binding to DNA and SRF, the Elk-1 mutant REST/Elk-1 $\Delta \mathrm{C}$ most likely also inhibits the activity of two other ternary complex factors, SAP-1 and SAP-2. These experiments revealed that expression of REST/Elk-1 $\triangle \mathrm{C}$ completely blocked the stimulus-induced biosynthesis of Egr-1 in cerulein stimulated AR42J cells as well as in CNO-stimulated AR42J cells expressing Gaq-coupled designer receptors. Thus, ternary complex factor activation is a key step in connecting Gaq-stimulation with enhanced Egr-1 biosynthesis.

The induction of acute pancreatitis via injection of cerulein into mice is a frequently used model system of this disease. Several observations suggest that activation of the proinflammatory transcription factor Egr-1 is required to induce the inflammatory response following induction of acute pancreatitis. Using the AR42J cellular model, we identified the signaling molecules $\mathrm{Ca}^{2+}$, Raf, ERK, MKP-1, and TCF that connect cerulein stimulation with enhanced expression of Egr-1. These molecules represent new targets for pharmacological intervention in pancreatitis. The essential role of $\mathrm{Ca}^{2+}$ ions, and the functions of ERK and MKP-1 have been discussed in the framework of cerulein-induced pancreatitis [6, 51-54]. Raf and TCFs have not yet been the focus of studies of the cerulein-induced signaling cascade leading to an acute pancreatitis. Future experiments, involving mouse models, will clarify the role of these signaling molecules in healthy and dysfunctional pancreatic acinar cells.

\section{Abbreviations}

CCK (cholecystokinin); CNO (clozapine-N-oxide); Egr (early growth response); ERK (extracellular signal-regulated protein kinase); MKP (MAP kinase phosphatase); PKC (protein kinase C); TCF (ternary complex factor).

\section{Disclosure Statement}

The authors declare that there is no conflict of interest.

\section{Acknowledgement}

We thank Bernd Baumann for AR42J cells and Libby Guethlein for critical reading of the manuscript. 


\section{References}

1 Kim H: Cerulein pancreatitis: Oxidative stress, inflammation, and apoptosis. Gut and Liver 2008;2:74-80.

2 Frossard JL, Steer M, Pastor CM: 2008. Acute pancreatitis. Lancet 2008;371:143-152.

-3 Gukovskaya AS, Gukovsky I, Zaninovic V, Song M, Sandoval D, Gukovsky S: Pancreatic acinar cells produce, release, and respond to tumor necrosis factor- $\alpha$. Role in regulating cell death and pancreatitis. J Clin Invest 1997;100:1853-1862.

- 4 Frossard JL, Saluja A, Bhagat L, Lee HS, Bhatia M, Hofbauer B, Steer ML: The role of intracellular adhesion molecule-1 and neutrophils in acute pancreatitis and pancreatitis-associated lung injury. Gastroenterology 1999;116:694-701.

5 Pastor CM, Rubbia-Brandt L, Hadengue A, Jordan M, Morel P, Frossard JL: Role of macrophage inflammatory peptide- 2 in cerulein-induced acute pancreatitis and pancreatitis-associated lung injury. Lab Invest 2002;83:471-478.

-6 Sendler M, Dummer A, Weiss FU, Krüger B, Wartmann T, Scharffetter-Kochanek K, van Rooijen N, Malla SR, Aghdassi A, Halangk W, Lerch MM, Mayerle J: Tumor necrosis factor $\alpha$ secretion induces protease activation and acinar cell necrosis in acute experimental pancreatitis in mice. Gut 2013;62:430-439.

7 Maltzman JS, Carman JA, Monroe JG: Transcriptional regulation of the Icam-1 gene in antigen receptor- and phorbol ester-stimulated B lymphocytes: Role for transcription factor EGR1. J Exp Med 1996;183:17471759.

8 Yao J, Mackman N, Edgington TS, Fan S-T: Lipopolysaccharide induction of the tumor necrosis factor- $\alpha$

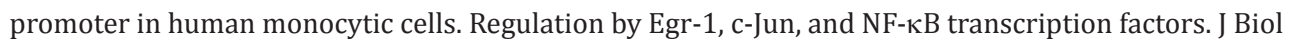
Chem 1997;272:17795-17801.

-9 Yan SF, Fujita T, Lu K, Okada K, Zou YS, Mackman N, Pinsky DJ, Stern DM: Egr-1, a master switch coordinating upregulation of divergent gene families underlying ischemic stress. Nat Med 2000;6:13551361.

10 Silverman ES., De Sanctis GT, Boyce J, Maclean JA, Jiao A, Green FH, Grasemann H, Faunce D, Fitzmaurice G, Shi GP, Stein-Streilein J, Milbrandt J, Collins T, Drazen JM: The transcription factor early growth response factor 1 modulates tumor necrosis factor- $\alpha$, immunoglobulin $\mathrm{E}$, and airway responsivness in mice. Am J Respir Crit Care Med 2001;163:778-785.

11 Ji B, Chen X-q, Misek DE, Kuick R, Hanash S, Ernst S, Najarian R, Logsdon CD: Pancreatic gene expression during initiation of acute pancreatitis: identification of EGR-1 as a key regulator. Physiol Genom 2003;14:59-72.

12 Pritchard MT, Roychowdhury S, McMullen MR, Guo L, Arteel GE, Nagy LE: Early growth response-1 contributes to galactosamine/lipopolysaccharide-induced acute liver injury in mice. Am J Physiol Gastrointest Liver Physiol 2007;293:G1124-G1133.

-13 Sandoval J, Pereda J, Rodriguez JL, Escobar J, Hidalgo J, Joosten LAB, Franco L, Sastre J, López-Rodas G: Ordered transcriptional factor recruitment and epigenetic regulation of tnf- $\alpha$ in necrotizing acute pancreatitis. Cell Mol Life Sci 2010:67:1687-1697.

14 Wan H., Yuan Y, Liu J, Chen G: Pioglitazone, a PPAR- $\gamma$ activator, attenuates the severity of cerulein-induced acute pancreatitis by modulating early growth response-1 transcription factor. Transl Res 2012;160:153161.

15 Thiel G, Cibelli G: Regulation of life and death by the zinc finger transcription factor Egr-1. J Cell Physiol 2002;193:287-292.

16 Rössler OG, Stefano L, Bauer I, Thiel G: Stimulus-transcription coupling in the nervous system - The zinc finger protein Egr-1; in Thiel G (ed): Transcription factors in the nervous system - Development, brain function, and disease. Wiley-VCH, Weinheim, Germany, 2006, pp 379-395.

17 Gong LB, Liu Y, Chen X-Q, Jiang B: Expression of early growth response factor-1 in rats with ceruleininduced acute pancreatitis and its significance. World J Gastroenterol 2005;11:5022-5024.

18 McMahon M: Steroid receptor fusion proteins for conditional activation of Raf-MEK-ERK signaling pathway. Meth Enzymol 2001;332:401-417.

19 Rössler OG, Giehl KM, Thiel G: Neuroprotection of immortalized hippocampal neurons by brain-derived neurotrophic factor and Raf-1 protein kinase: Role of extracellular signal-regulated protein kinase and phosphatidylinositol 3-kinase. J Neurochem 2004;88:1240-1252. 
20 Thiel G, Rössler OG: Immediate-early transcriptional response to angiotensin II in human adrenocortical cells. Endocrinology 2011;152:4211-4223.

21 Thiel G, Lesch A, Keim A: Transcriptional response to calcium-sensing receptor stimulation. Endocrinology 2012;153:4716-4728.

22 Kaufmann A, Keim A, Thiel G: Regulation of immediate-early gene transcription following activation of Gaq-coupled designer receptors. J Cell Biochem 2013;114:681-696.

-23 Spohn D, Rössler OG, Philipp SE, Raubuch M, Kitajima S, Griesemer D, Hoth M, Thiel G: Thapsigargin induces expression of activating transcription factor 3 in human keratinocytes involving $\mathrm{Ca}^{2+}$ ions and c-Jun N-terminal protein kinase. Mol Pharmacol 2010;78:865-876.

24 Keim A, Müller I, Thiel G: Efficient genetic manipulation of 1321N1 astrocytoma cells using lentiviral gene transfer. J Neurosci Meth 2012;206:138-142.

25 Kaufmann K, Thiel G: Epidermal growth factor and thrombin induced proliferation of immortalized human keratinocytes is coupled to the synthesis of Egr-1, a zinc finger transcriptional regulator. J Cell Biochem 2002;85:381-391.

26 Mayer SI, Müller I, Mannebach S, Endo T, Thiel G: Signal transduction of pregnenolone sulfate in insulinoma cells. Activation of Egr-1 expression involving TRPM3, voltage-gated calcium channels, ERK, and ternary complex factors. J Biol Chem 2011;286:10084-10096.

27 Piiper A, Elez R, You S-J, Kronenberger B, Loitsch S, Roche S, Zeuzem S: Cholecystokinin stimulates extracellular signal-regulated protein kinase through activation of the epidermal growth factor receptor, Yes, and protein kinase C. J Biol Chem 2003;278:7065-7072.

-28 Duan WR, Ito M, Park Y, Maizels ET, Hunzicker-Dunn M, Jameson JL: GnRH regulates early growth response protein 1 transcription through multiple promoter elements. Mol Endocrinol 2002;16:221-233.

29 Rössler OG, Thiel G: Thrombin induces Egr-1 expression in fibroblasts involving elevation of the intracellular $\mathrm{Ca}^{2+}$ concentration, phosphorylation of ERK and activation of ternary complex factor. BMC Mol Biol 2009;10:40.

30 Kaufmann K, Bach K, Thiel G: Extracellular signal-regulated protein kinases Erk1/Erk2 stimulate expression and biological activity of the transcriptional regulator Egr-1. Biol Chem 2001;382:1077-1081.

-31 Mayer SI, Willars GB. Nishida E, Thiel G: Elk-1, CREB, and MKP-1 regulate Egr-1 expression in gonadotropin-releasing hormone stimulated gonadotrophs. J Cell Biochem 2008;105:1267-1278.

-32 Mayer SI, Thiel G: Calcium influx into MIN6 insulinoma cells induces expression of Egr-1 involving extracellular signal-regulated protein kinase and the transcription factors Elk-1 and CREB. Eur J Cell Biol 2009;88:19-33.

-33 Dabrowski A, Groblewski GE, Schäfer C, Guan K-L, Williams JA: Cholecystokinin and EGF activate a MAPK cascade by different mechanisms in rat pancreatic acinar cells. Am J Physiol - Cell Physiol 2007;273:C1472-C1479.

34 Thiel G, Ekici M, Rössler OG: Regulation of cellular proliferation, differentiation and cell death by activated Raf. Cell Commun Signal 2009;7:8.

35 Yokoyama T, Takano K, Yoshida A, Katada F, Sun P, Takenawa T, Andoh T, Endo T: DA-Raf1, a competent intrinsic dominant-negative antagonist of the Ras-ERK pathway, is required for myogenic differentiation. J Cell Biol 2007;177:781-793.

-36 Dudley DT, Pang L, Decker SJ, Bridges AJ, and Saltiel AR: A synthetic inhibitor of the mitogen-activated protein kinase cascade. Proc Natl Acad Sci USA 1995;92:7686-7689.

- 37 Stefano L, Rössler OG, Griesemer D, Hoth M, Thiel G: P2X receptor stimulation upregulates Egr-1 biosynthesis involving a cytosolic $\mathrm{Ca}^{2+}$ rise, transactivation of the EGF receptor and phosphorylation of ERK and Elk-1. J Cell Physiol 2007;213:36-44.

- 38 Mayer SI, Rössler OG, Endo T, Charnay P, Thiel G: Epidermal growth factor-induced proliferation of astrocytes requires Egr transcription factors. J Cell Sci 2009;122:3340-3350.

-39 Cesari F, Brecht S, Vintersten K, Vuong LG, Hofmann M, Klingel K, Schnorr J-J, Arsenian S, Schild H, Herdegen T, Wiebel FF, Nordheim A: Mice deficient for the Ets transcription factor Elk-1 show normal immune responses and mildly impaired neuronal gene activation. Mol Cell Biol 2004;24:294-305.

-40 Thiel G, Lietz M, Cramer, M: Biological activity and modular structure of RE-1 silencing transcription factor (REST), a repressor of neuronal genes. J Biol Chem 1998;273:26891-26899.

41 Dufresne M, Seva C, Fourmy D: Cholecystokinin and gastrin receptors. Physiol Rev 2006;86:805-847. 
Kaufmann/Rössler/Thiel: Egr-1 Expression in the Pancreas

42 Xiao RP, Zhu W, Zheng M, Cao C, Zhang Y, Lakatta EG, Han Q: Subtype-specific $\mathrm{a}_{1}$ - and b-adrenoceptor signaling in the heart. Trends Pharmacol Sci 2006;27:330-337.

43 Dobkin-Bekman M, Naidich M, Pawson AJ, Millar RP, Seger R, Naor Z: Activation of mitogen-activated protein kinase (MAPK) by GnRH is cell-context dependent. Mol Cell Endocrinol 2006;252:184-190.

$\checkmark 44$ Lee SY, Földy C, Szabadics J, Soltesz I: Cell-type specific CCK2 receptor signaling underlies the colecytokinin-mediated selective excitation of hippocampal parvalbumin-positive fast-spiking basket cells. J Neurosci 2011;31:10993-11002.

45 Thiel G, Kaufmann A, Rössler OG: G-protein coupled designer receptors - new chemical-genetic tools for signal transduction research. Biol Chem 2013;394:1615-1622.

46 Wess J, Nakajima K, Jain S: Novel designer receptors to probe GPCR signaling and physiology. Trends Pharmacol Sci 2013;34:385-392.

47 Rössler OG, Henß I, Thiel G: Transcriptional response to muscarinic acetylcholine receptor stimulation: Regulation of Egr-1 biosynthesis by Elk-1, ERK, MKP-1 and calcineurin in carbachol stimulated human neuroblastoma cells. Arch Biochem Biophys 2008;470:93-102.

48 Müller I, Endo T, Thiel G: Regulation of AP-1 activity in glucose-stimulated insulinoma cells. J Cell Biochem 2010;110:1481-1494.

49 Ayadi AH, Zheng H, Sobieszczuk P, Buchwalter G, Moerman P, Alitalo K, Wasylyk B: Net-targeted mutant mice develop a vascular phenotype and up-regulate egr-1. EMBO J 2001;20:5139-5152.

50 Costello PS, Nicolas RH, Watanabe Y, Rosewell I, Treisman R: Ternary complex factor SAP-1 is required for Elk-mediated thymocyte positive selection. Nat Immunol 2004;5:289-298.

-51 Krüger B, Albrecht E, Lerch MM: The role of intracellular calcium signaling in premature protease activation and the onset of pancreatitis. Am J Pathol 2000;157:43-50.

-52 Namkung W, Yoon JS, Kim KH, Lee MG: PAR2 exerts local protection against acute panceatitis via modulation of MAP kinase and MAP kinase phosphatase signaling. Am J Physiol - Gastrointest Liver Physiol 2008;295:G886-G894.

-53 Kim MS, Hong JH, Li Q, Shin DM, Abramowitz J, Birnbaumer L, Muallem S: Deletion of TRPC3 in mice reduces store-operated $\mathrm{Ca}^{2+}$ influx and the severity of acute pancreatitis. Gastroenterology 2009;137:15091517.

-54 Mazzon E, Impellizzeri D, Di Paola R, Paterniti I, Esposito E, Cappellani A, Bramanti P, Cuzzocrea S: Effects of mitogen-activated protein kinase signaling pathway inhibition on the development of cerulein-induced acute pancreatitis in mice. Pancreas 2012;41:560-570. 